\title{
Towards metal chalcogenide nanowire-based colour-sensitive photodetectors
}

\author{
Edgars Butanovs ${ }^{*}$, Jelena Butikova, Aleksejs Zolotarjovs, Boris Polyakov \\ Institute of Solid State Physics, University of Latvia, Kengaraga Street 8, Riga, LV-1063, Latvia
}

a bstract

In recent years, nanowires have been shown to exhibit high photosensitivities, and, therefore are of interest in a variety of optoelectronic applications, for example, colour-sensitive photodetectors. In this study, we fabricated two-terminal PbS,

$\mathrm{In}_{2} \mathrm{~S}_{3}$, CdS and $\mathrm{ZnSe}$ single-nanowire photoresistor devices and tested applicability of these materials under the same conditions for colour-sensitive ( $405 \mathrm{~nm}, 532 \mathrm{~nm}$ and $660 \mathrm{~nm}$ ) light detection. Nanowires were grown via atmospheric pressure chemical vapour transport method, their structure and morphology were characterized by scanning and transmission electron microscopy (SEM and TEM), X-ray diffraction (XRD), and optical properties were investigated with photoluminescence (PL) measurements. Single-nanowire photoresistors were fabricated via in situ nanomanipulations inside SEM, using focused ion beam (FIB) cutting and electron-beam-assisted plat-inum welding; their current-voltage characteristics and photoresponse values were measured. Appli-cability of the tested nanowire materials for colour-sensitive light detection is discussed.

\section{Introduction}

Colour-sensitive photodetectors are desirable for a large variety of applications, for instance, in optical communications, digital imaging and environmental monitoring [1e4]. In recent years, nanowires (NWs) have been demonstrated to be superior to their bulk counterparts as photodetector materials mainly due to their higher sensitivities, which arise from the NW high surface area/ volume ratio resulting in high density of surface states that act as trap sites for photogenerated holes, therefore increasing electron lifetime and enhancing the photocurrent [5,6]. Semiconducting chalcogenide materials with direct bandgap and large absorption coefficients, such as $\mathrm{PbS}$,

$\mathrm{In}_{2} \mathrm{~S}_{3}, \mathrm{CdS}, \mathrm{ZnSe}$, are great candidates for next-generation NW-based light detectors due to their excellent photodetection properties [6e9]. Typical photodetectors can detect only a specific spectral band, therefore are not suitable for multi-colour detection with spectral selectivity. There are two general approaches of making colour-sensitive detectors: using broadspectrum materials and wavelength-selective filters, or combining several materials with different bandgaps, including material doping and composition tuning. Using broad-spectrum could be possibly used to fabricate multi-colour photode-tectors [13]. Sang et al. utilized CdS, $\mathrm{SnO}_{2}, \mathrm{ZnO}$ and $\mathrm{Ga}_{2} \mathrm{O}_{3}$ one-dimensional nanostructures to demonstrate colour-sensitive pho-todetection by simultaneously integrating them into an insulating substrate with a two-terminal device structure [14]. In such a way, the cut-off wavelengths of the selected semiconductor NW materials, defined by the bandgap, will determine the spectral selec-tivity. This approach benefits from substantial freedom to choose NW materials with desirable spectral band and high photodetector materials combined with absorptive colour filters is a possibility; however, it involves additional fabrication steps and, to obtain highest efficiency, one would prefer light to be converted to photocurrent rather than reflected or absorbed in the filter layer. Research has been done on compositionally tuneable NWs and doping of NWs for wavelength-selective light detection $[10,11]$, but there is a difficulty to extend the optical band outside the visible light range. Furthermore, it has been demonstrated that colour imaging is possible by varying the radius of silicon NWs to control spectral sensitivities [12]. However, a versatile strategy to obtain spectral selectivity is to select arbitrary photosensitive ma-terial NWs with different bandgaps, and incorporate them in a composite thin film or on an insulating substrate. Liu et al. demonstrated a method of incorporating photosensitive $\mathrm{CdS}, \mathrm{CdSeS}$ and CdSe NWs into an amorphous indium zinc oxide thin film to obtain a transparent thin-film-transistor array, showing that such concept 
photoresponse. Furthermore, large-scale printing of NWs at defined locations on flexible substrates via roll-to-roll transfer has been demonstrated [15], thus enabling the development of NW based all-printed electronic and optoelectronic devices at low cost and with high performance.

In this work, we synthesised $\mathrm{PbS}, \mathrm{In}_{2} \mathrm{~S}_{3}, \mathrm{CdS}$ and $\mathrm{ZnSe} \mathrm{NWs}$ and fabricated single-nanowire two-terminal photoresistor devices to test the applicability of these materials for colour-sensitive (red, green and blue light) photodetector applications, if they would be simultaneously incorporated on the same substrate. It is important to note that all these devices were prepared using the same method and tested under the same conditions. The measured NW photo-detector properties were compared and analysed.

\section{Experimental details}

\subsection{Nanowire synthesis and characterization}

The following material NWs were synthesised via atmospheric pressure chemical vapour transport method in a horizontal quartz tube reactor by adjusting previously reported growth parameters: $\mathrm{PbS}$ [16], In2 $\mathrm{S}_{3}$ [17], CdS [18], and ZnSe [19]. In all the cases, the NWs were grown on oxidized silicon wafers $\mathrm{Si}(110) / \mathrm{SiO}_{2}$ (Semiconductor Wafer, Inc.) coated with spherical Au nanoparticles used as a cata-lyst (Smart materials, water suspension, $50 \mathrm{~nm}$ diameter).

PbS NWs: $0.25 \mathrm{~g} \mathrm{PbCl}_{2}$ powder (98\%, Sigma Aldrich) was loaded in a ceramic boat and placed in the centre of the quartz tube at $650 \mathrm{C}, \mathrm{Au} / \mathrm{Si}$ substrate was placed downstream in a lower temperature region. Excessive amount of sulphur powder (enola SIA) was placed upstream at $250 \mathrm{C}$ to create sulphur-rich atmosphere, while $\mathrm{N}_{2}$ was used as a carrier gas. The temperature was kept constant for $20 \mathrm{~min}$, followed by natural cooling to room temperature.

$\mathrm{In}_{2} \mathrm{~S}_{3} \mathrm{NWs}$ : Mixture of $0.5 \mathrm{~g}$ In and $0.5 \mathrm{~g} \mathrm{InCl}_{3}$ (98\%, Sigma-Aldrich) powders were used as a source material and sent to the centre of the quartz tube. $\mathrm{Au} / \mathrm{Si}$ substrate and sulphur powder were placed downstream and upstream, respectively, as in the previous case. $\mathrm{Ar} / \mathrm{H}_{2}(5 \%)$ gas mixture was used as a vapour carrier. The furnace was heated to $800 \mathrm{C}$, temperature was held constant for
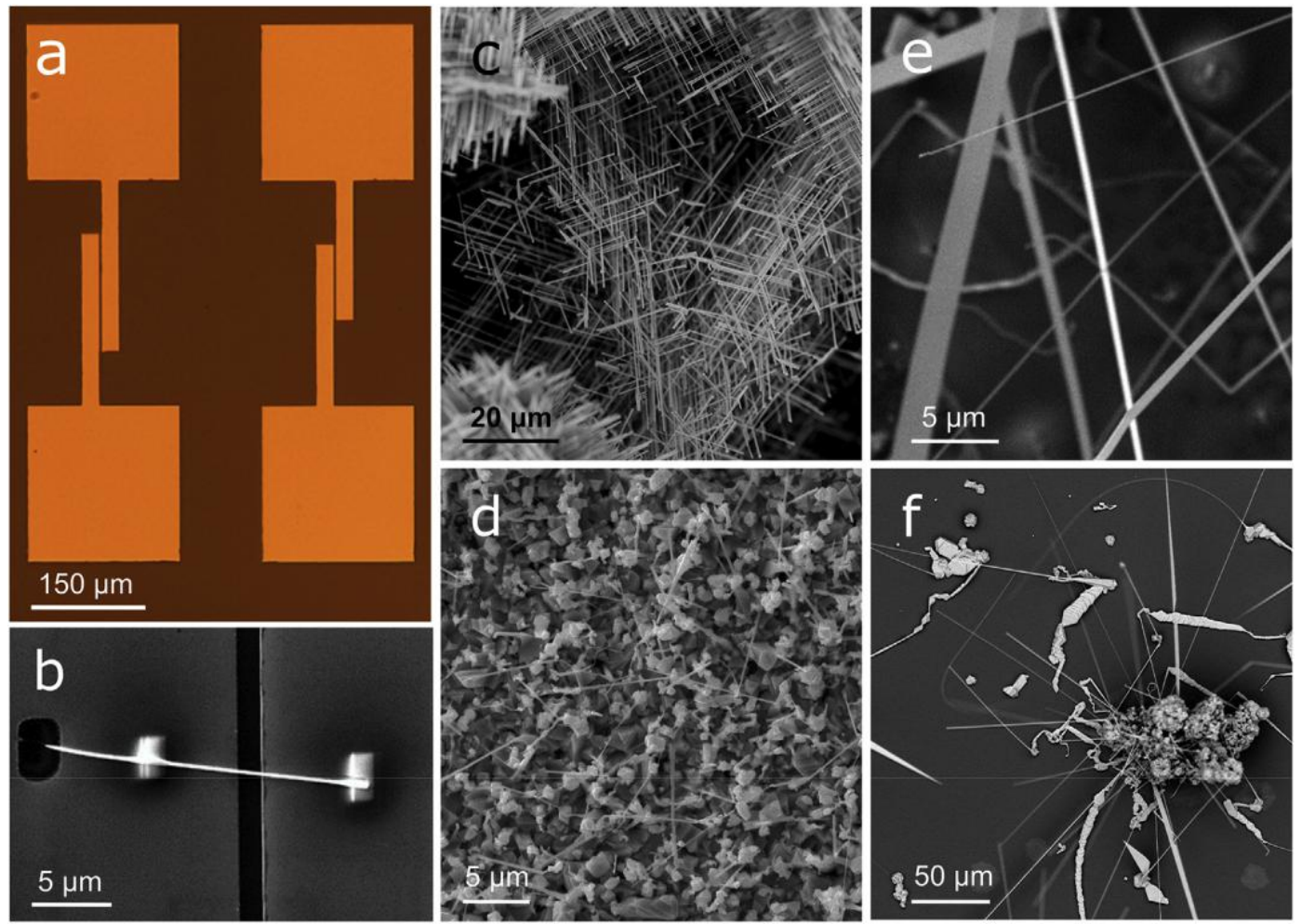

Fig. 1. (a) Optical microscope image of gold microelectrodes on the oxidized silicon substrate; SEM images of (b) a typical as-prepared nanowire photoresistor; as-grown (c) PbS, (d) In2S3, (e) CdS and (f) ZnSe nanowires.
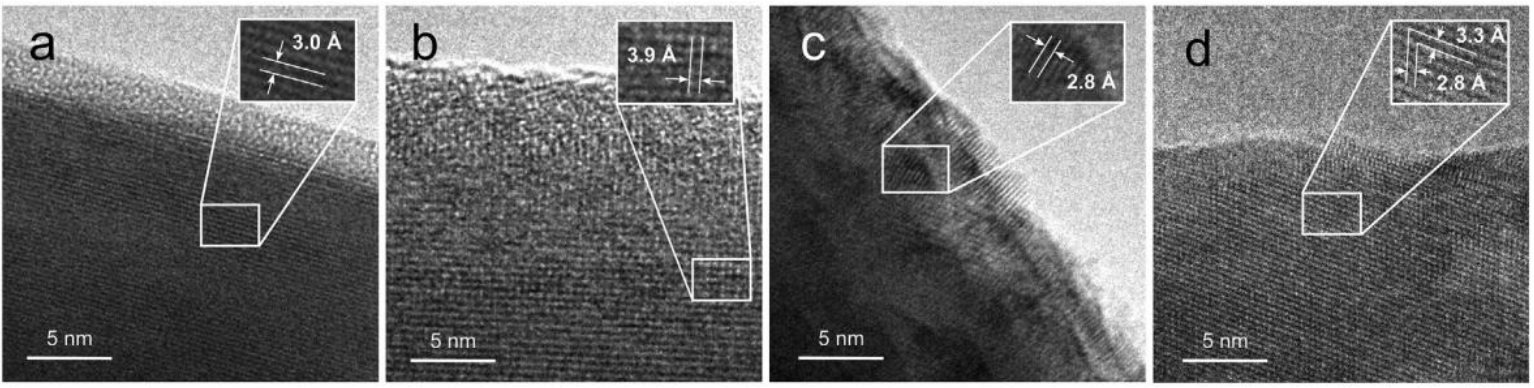

Fig. 2. TEM images of (a) $\mathrm{PbS}$, (b) In2S3, (c) CdS and (d) ZnSe nanowires. Insets show measured atomic interlayer distances. 
$45 \mathrm{~min}$ and then was let to cool down naturally to room temperature.

CdS NWs: CdS powder (98\%, Alfa Aesar) was thermally evapo-rated in a quartz tube at $950 \mathrm{C}$ temperature for $30 \mathrm{~min}$, followed by a natural cooling. The vapour was carried downstream to the $\mathrm{Au} / \mathrm{Si}$ substrate by $\mathrm{N}_{2}$ gas.

ZnSe NWs: $0.2 \mathrm{~g}$ home-made ZnSe powder was placed at the centre of the quartz tube and evaporated at $1000 \mathrm{C}$ for $2.5 \mathrm{~h}$, using $\mathrm{Ar} / \mathrm{H}_{2}(5 \%)$ gas to carry vapour downstream to the $\mathrm{Au} / \mathrm{Si}$ substrate at a lower temperature region, and then cooled down naturally to the room temperature.

As-prepared NW morphology was characterized by scanning electron microscopy (SEM, Lyra, Tescan), whereas the inner crys-talline structure was revealed by transmission electron microscopy (TEM, Tecnai GF20, FEI) using the operating voltage of $200 \mathrm{kV}$. X-ray diffraction (XRD, PANalytical, $X^{\prime}$ Pert Pro Powder) was carried out by monochromatic $\mathrm{Cu} \mathrm{Ka}$ irradiation to confirm the material and the phase of a NW. Room-temperature photoluminescence (PL, Hamamatsu R92P PMT) spectra with the excitation wavelength of $266 \mathrm{~nm}$ (fourth harmonic of Nd:YAG laser) for all NWs and infrared (IR) absorption spectrum (Bruker Equinox 55 FT-IR Spectrometer) for $\mathrm{PbS}$ NWs were measured to investigate their optical properties.

\subsection{Single nanowire photoresistor fabrication}

Firstly, gold microelectrodes with the gap width of $2 \mathrm{~mm}$ were prepared on an oxidized silicon wafer by conventional photoli-thography technique (see Fig. 1(a)). Briefly, microelectrode pattern was obtained using direct write laser lithography (mPG 101, Hei-delberg Instruments) on SU-8 2003 photoresist (MicroChem), 5/ $45 \mathrm{~nm} \mathrm{Cr/Au} \mathrm{film} \mathrm{was} \mathrm{deposited} \mathrm{via} \mathrm{thermal}$ evaporation method, followed by a lift-off procedure.

Secondly, single NW photoresistors were fabricated using in situ nanomanipulations inside SEM-FIB. As-grown NWs were me-chanically transferred from the Si substrate to the as-prepared gold microelectrodes by welding a single NW to the tungsten (W) nanomanipulator probe using electron-beam-assisted platinum $(\mathrm{Pt})$ deposition. After aligning and placing it on top of the micro-electrodes, NW was cut off from the W probe with gallium $(\mathrm{Ga})$ ion beam and welded to the electrodes with $\mathrm{Pt}$ deposition to ensure the electric contact and fixed position. SEM image of a typical asprepared photoresistor is displayed in Fig. 1(b), where the narrow dark strip is the gap between the electrodes, two small rectangles on the NW are the deposited Pt contacts, and the trench appeared due to the cutting NW by ion beam milling.

\subsection{Device measurements}

Current-voltage $\left(\mathrm{I}_{\mathrm{ds}}-\mathrm{V}_{\mathrm{ds}}\right)$ characteristics and photoresponse of the fabricated two-terminal devices were measured at zero gate voltage $\left(\mathrm{V}_{\mathrm{g}}{ }^{1 / 4} 0\right.$ V) with a two-contact micro probe station con-nected with Model 6485 Keithley Picoammeter, Model 2000 Keithley Multimeter and a voltage source (33220A Waveform Generator, Agilent). $405 \mathrm{~nm}, 532 \mathrm{~nm}$ and $660 \mathrm{~nm}$ wavelength semiconductor diode lasers with light intensity of $2 \mathrm{~W} / \mathrm{cm}^{2}$ were used as an illumination source for the photoresponse measure-ments. All measurements were carried out in air and at room temperature.

\section{Results and discussion}

\subsection{Morphology, structure and PL measurements}

SEM was used to image as-grown NW arrays (see Fig. 1(cef)) and to determine the size of individual NWs. The length varied from 20 to $30 \mathrm{~mm}$ for $\mathrm{PbS}$ and $\mathrm{In}_{2} \mathrm{~S}_{3} \mathrm{NWs}$ to several hundred micrometres for CdS and ZnSe NWs. The diameter of NWs was in the range of 50e300 $\mathrm{nm}$. The Au catalyst nanoparticles were observed on the top end of $\operatorname{In}_{2} \mathrm{~S}_{3}$, CdS and ZnSe NWs, which in-dicates Vapour-Liquid-Solid (VLS) growth, as was expected [20e22]; however, that was not the case for PbS NWs: these NWs exhibited growth in hierarchical, orthogonally branched clusters, as reported previously [23]. It is worth noting that nanobelts were also observed on as-prepared $\mathrm{CdS}$ and $\mathrm{ZnSe}$ samples, along with the NWs, though only NWs were used to fabricate photoresistor

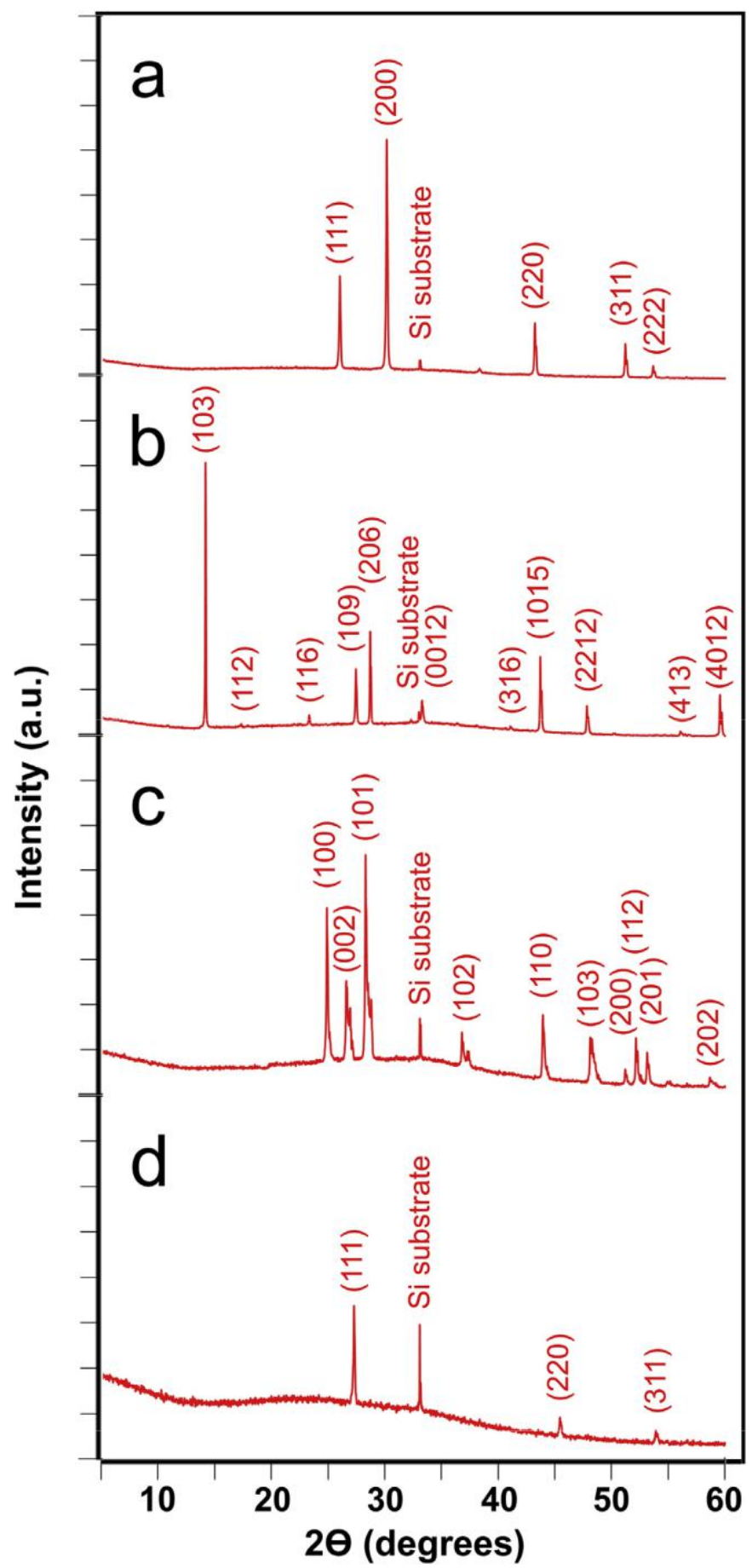

Fig. 3. XRD spectra of (a) PbS, (b) In2S3, (c) $\mathrm{CdS}$ and (d) ZnSe nanowire arrays on a silicon substrate. 
devices.

TEM investigations revealed inner crystalline structure of the NWs. Fig. 2(a) shows highly crystalline nature of a PbS NW. Resolved atomic planes are separated by a distance of $3.0 \AA$, which corresponds to (200) planar spacing of a cubic (rock-salt) PbS crystal (a 1/4 $5.94 \AA$ [24]). Similarly, an In $2 S_{3}$ NW exhibited high crystallinity, as can be seen in Fig. 2(b). Interplanar spacing was measured to be $3.9 \AA$, which is in a good agreement with the lattice constant of tetragonal b-In2 $\mathrm{S}_{3}$ (a $1 / 47.8 \AA$ [25]). Polycrystalline shell was observed around the CdS NW, which is visible in Fig. 2(c). According to interplanar distance measurements, such crystallites have cubic (zinc blende) structure with planar spacing of $2.7 \mathrm{e} 2.8 \AA$, which corresponds to the lattice constant value a $1 / 45.45 \AA$ [26]. As can be seen in Fig. 2(d), the distances between adjacent $\mathrm{ZnSe}$ lattice planes was measured to be $3.3 \AA$ and $2.8 \AA$, which corresponds to cubic (zinc blende) structure (a 1/4 $5.6 \AA$ [27]).

Fig. 3 depicts the XRD patterns of the as-grown NW arrays, which verifies their material and phase. The diffraction peaks in PbS NW XRD pattern were indexed to a cubic $\mathrm{PbS}$ crystal [24], In2S3 NW pattern to a tetragonal b-In2S3 crystal [25], CdS NW pattern to a hexagonal wurtzite structure [28], and ZnSe XRD pattern was indexed to cubic (zinc blende) $\mathrm{ZnSe}$ crystal structure [27]. The peak at 33 is attributed to the diffraction in the Si substrate.

PL properties of the as-grown NWs were studied at the room temperature in the wavelength range from $380 \mathrm{~nm}$ to $580 \mathrm{~nm}$, excited by $266 \mathrm{~nm}$ laser. Measured spectra are shown in Fig. 4. The bandgap of PbS NWs is around 0.4 $\mathrm{eV}$ and the optical absorption starts at $3100 \mathrm{~nm}$, therefore luminescence band with maximum at $425 \mathrm{~nm}$ (see Fig. 4(a)) corresponds to higher energy band transi-tions $[29,30]$. Similar luminescence was measured on $\mathrm{PbS}$ nano-cubes and thin films in other works by Cao et al. [31] and Tohidi
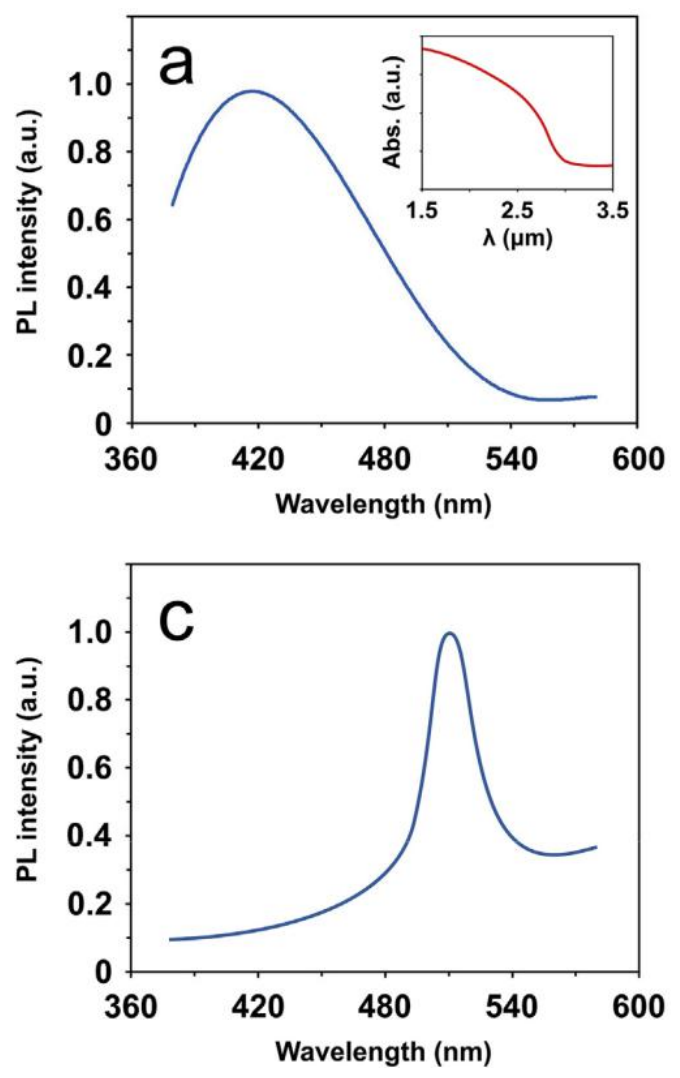

et al. [32]. The inset of Fig. 4(a) depicts measured optical absorption spectrum of $\mathrm{PbS} \mathrm{NWs}$, thus confirming that the absorption starts around $3 \mathrm{~mm}$ wavelength light. Fig. 4(b, c, d) shows measured luminescence bands of $\mathrm{In}_{2} \mathrm{~S}_{3}, \mathrm{CdS}$ and $\mathrm{ZnSe}$, respectively. Their ac-cording maxima are $505 \mathrm{~nm}, 510$ $\mathrm{nm}$ and $460 \mathrm{~nm}$, that corresponds to bandgap values of $2.45 \mathrm{eV}, 2.43 \mathrm{eV}$ and $2.7 \mathrm{eV}$, in agreement with previous reports $[6,19,20]$.

\subsection{Device photoresponse measurements}

Fig. 5 shows measured $\mathrm{I}_{\mathrm{ds}}-\mathrm{V}_{\mathrm{ds}}$ curves of different as-prepared twoterminal NW devices. At least four photoresistors of each material were fabricated, so consistent conclusions could be made. Typically, nearly symmetrical characteristics were measured for all investigated NW materials, therefore indicating that ohmic con-tacts were formed between the electrodes and the NW. Features of non-linear quadratic $\left(I \sim V^{2}\right)$ behaviour of the $I-V$ curves may be interpreted as an effect of the space-charge limited current (SCLC), as other groups have previously shown in different material nanowires [33,34]. In addition, it is worth noting that as-fabricated devices exhibit high resistance, wherein, most probably, a consid-erable part arises due to a high contact resistance since performed NW characterization indicated highly crystalline structure. Possible causes of such increased resistance include high resistance of deposited Pt contacts due to a carbon presence from the metal-organic precursor [35].

Next, as-prepared NW device electrical response to an illumi-nation of a light at different wavelengths was investigated. On-off photoresponse measurements, which are based on photoinduced conductivity changes, for three different illumination wavelengths at $\mathrm{V}_{\mathrm{ds}} 1 / 41 \mathrm{~V}$ bias of as-prepared NW photoresistor devices are
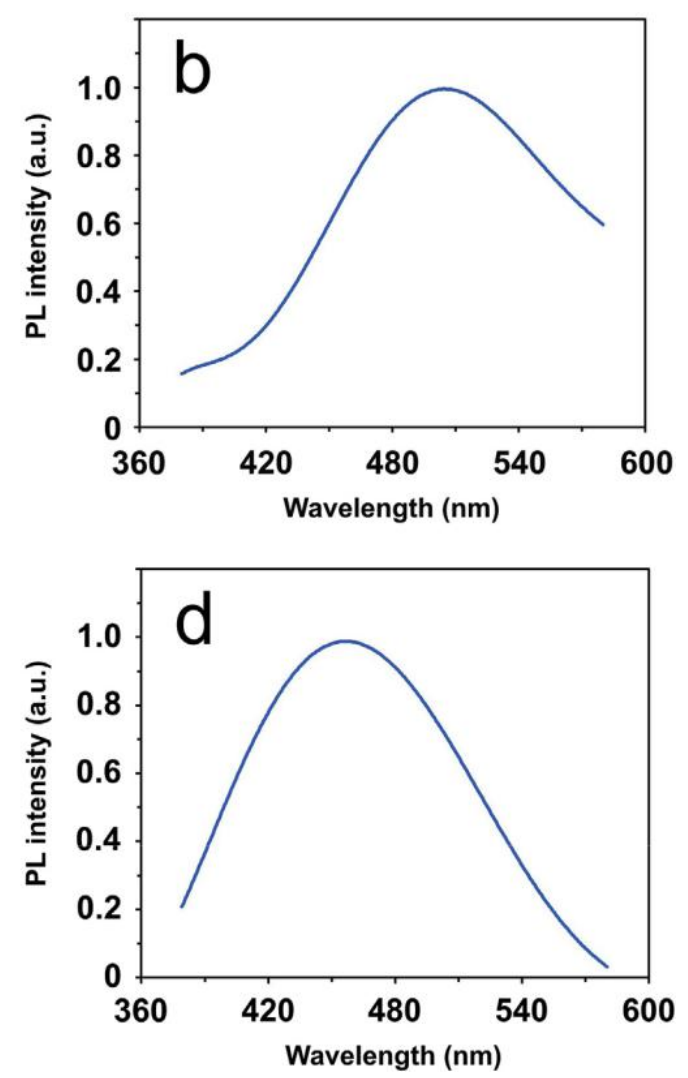

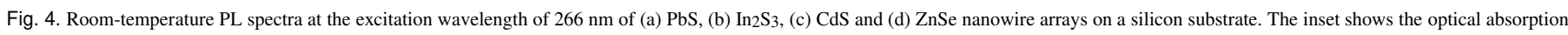
in $\mathrm{PbS}$ NWs in $1.5 \mathrm{e} 3.5 \mathrm{~mm}$ wavelength range. 

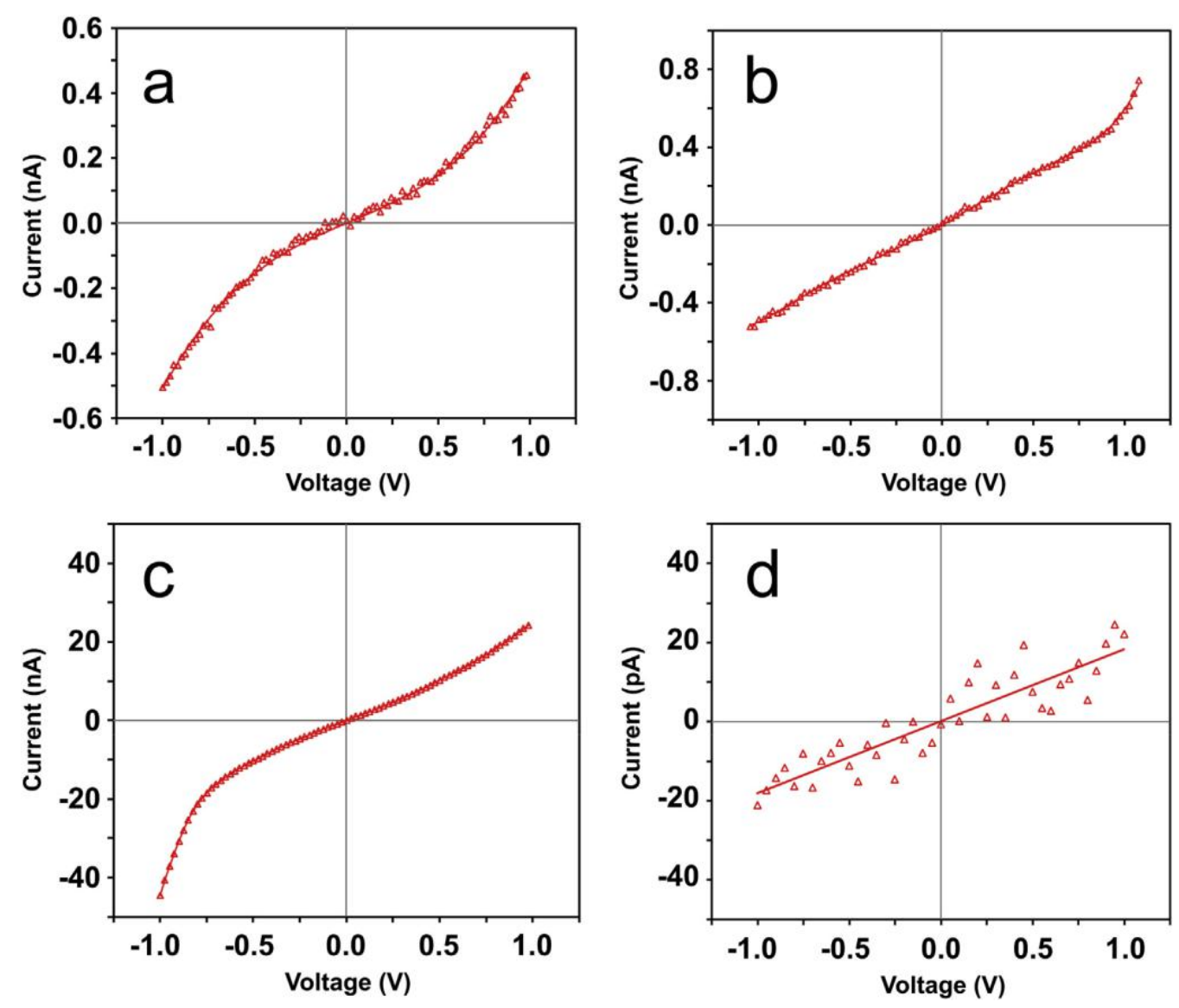

Fig. 5. Output (Ids- $\mathrm{V}_{\mathrm{ds}}$ ) characteristics at $\mathrm{Vg}_{\mathrm{g}} 1 / 40 \mathrm{~V}$ of (a) $\mathrm{PbS}$, (b) $\mathrm{In}_{2} \mathrm{~S}_{3}$, (c) $\mathrm{CdS}$ and (d) ZnSe nanowire photoresistors.

depicted in Fig. 6. It can be seen that all four studied NW materials exhibit rapid ( $<1 \mathrm{~s}$, in most cases) increase and decrease of the current after the illumination is turned on or off, respectively, except in the case of the $\operatorname{In}_{2} \mathrm{~S}_{3}$ NW current decay time for $405 \mathrm{~nm}$ illumination, which features a second, slower time component, most probably do to a presence of trapping centres.

Table 1 shows the comparison of current enhancement ratios $\left(\mathrm{I}_{\mathrm{on}} / \mathrm{I}_{\mathrm{off}}\right)$ of the studied NW materials in the context of their respective bandgap, which determines their cut-off wavelengths. Firstly, it can be seen that PbS NWs exhibit weak ( $\mathrm{I}_{\mathrm{on}} / \mathrm{I}_{\mathrm{off}}$ close to 1 ) photoresponse to all three illumination wavelengths; however, linear ratio vs. wavelength dependence was observed. Secondly, In2S3 and CdS NWs exhibit strong photoresponse to $405 \mathrm{~nm}$ illumination, and significantly weaker one to $532 \mathrm{~nm}$ and $660 \mathrm{~nm}$ illumination. Finally, ZnSe NWs show very strong response to $405 \mathrm{~nm}$ light, and no photoresponse was observed while illumi-nating them with $532 \mathrm{~nm}$ and 660

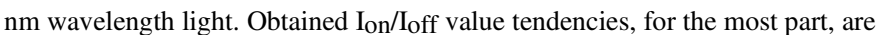
as was anticipated from the material bandgap values; however, relatively weaker photoresponse to $660 \mathrm{~nm}$ illumination was expected for either CdS or In2 $\mathrm{S}_{3} \mathrm{NWs}$.

Two important parameters for evaluating the quality of photo-conductors are the current responsivity (RI) and the external quantum efficiency (EQE). $\mathrm{R} \mid$ and EQE are defined, respectively, as R| 1/4 DI/(PS) and EQE 1/4 hcR //(el) [36], where DI is the difference between the photocurrent $I_{\text {on }}$ and the dark current $\mathrm{I}_{\mathrm{off}}, \mathrm{P}$ is the light power density, $\mathrm{S}$ is the effective illumination area (estimated as the electrode gap width NW diameter), $\mathrm{h}$ is Planck's constant, c is the speed of light, e is the electron charge, and 1 is the light wavelength. sensitivity. Table 2 contains the calculated $\mathrm{R} /$ and EQE values of the studied NW devices. The obtained data shows a relatively wide range of values for different NW materials, however these values are comparable to other typical state-of-art 1D nanostructure photodetectors [37], thus indicating the potential to use such ma-terials in future applications.

In principle, there are two approaches for determining the colour (red (660 $\mathrm{nm})$, green $(532 \mathrm{~nm})$ or blue $(405 \mathrm{~nm})$ ) of the incident light with such NW photoresistors, if they would be simultaneously incorporated on the same substrate as one opto-electronic device: by the wavelength cut-off due to the bandgap (signal or no signal) and by comparison of $\mathrm{I}_{\mathrm{on}} / \mathrm{I}_{\mathrm{off}}$ values of at least two different materials. In the perfect case, red, green and blue colours could be recognized by selecting three different materials with cut-off wavelengths in three different regions of the optical spectrum: one material that responds only to the blue light $\left(405 \mathrm{~nm}<\mathrm{I}_{\text {cut-off }}<532 \mathrm{~nm}\right)$, one that responds to the blue and green light $\left(532 \mathrm{~nm}<I_{\text {cut-off }}<660 \mathrm{~nm}\right)$, and one that responds to all three ( $\left.\right|_{\text {cut-off }}>660 \mathrm{~nm}$ ); and by illuminating them simulta-neously with the same light and comparing all photoresponse signals. However, in our case, only $\mathrm{ZnSe}$ measurement solely determined the incident (blue) light due to its cut-off wavelength. Other studied materials exhibited substantial decrease in photo-response in respect of the increasing wavelength, but still signifi-cant response was measured for the light with the longest wavelength, presumably due to some defect states, such as NW surface, or impurity doping. Thus, in such instance, one can only distinguish red and green or blue and green light of the same in-tensity by comparing absolute $\mathrm{I}_{\mathrm{on}} / \mathrm{I}_{\mathrm{off}}$ values of, for example, $\mathrm{PbS}$ and $\mathrm{CdS}$. Comparison could be made through logic operations of the 

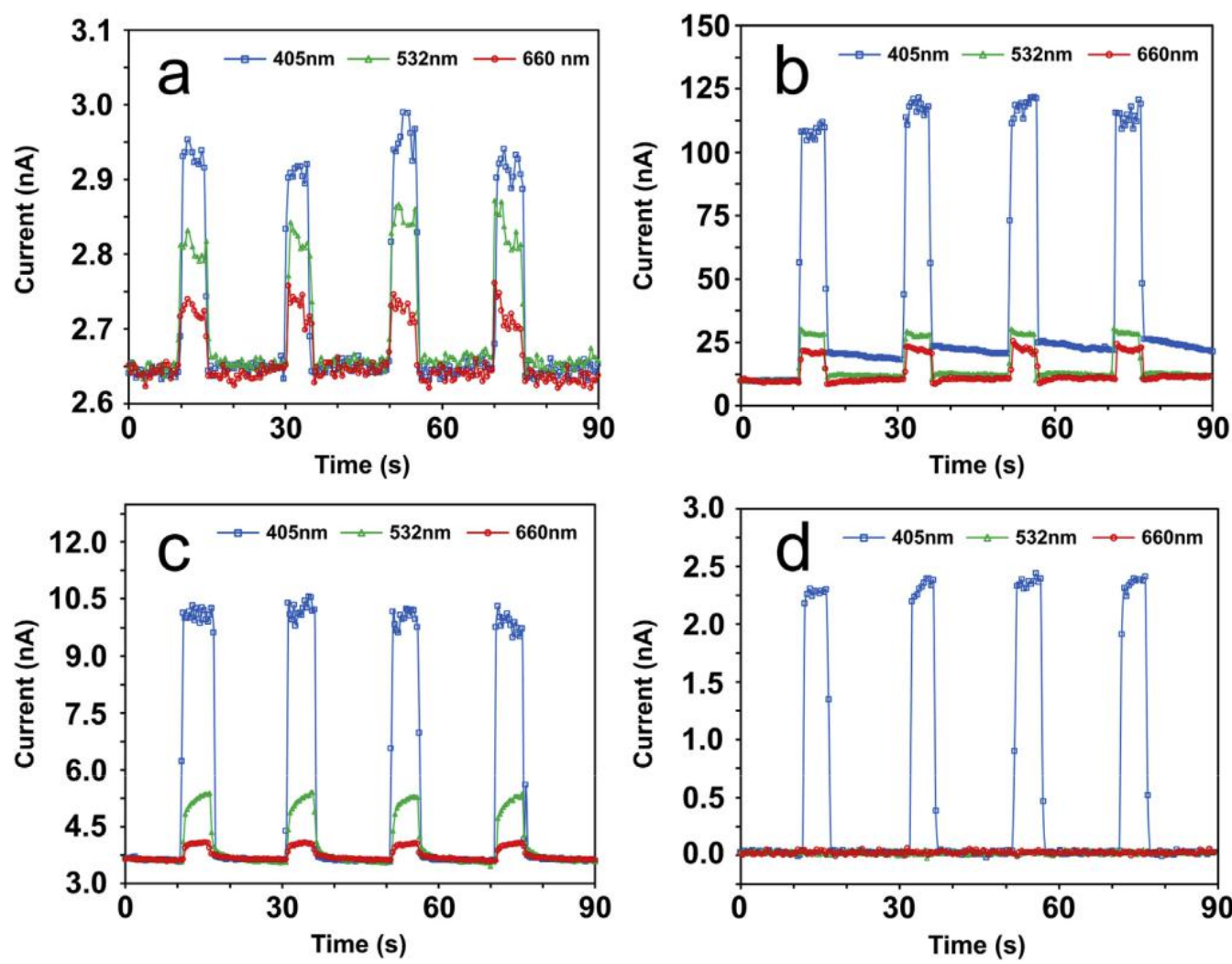

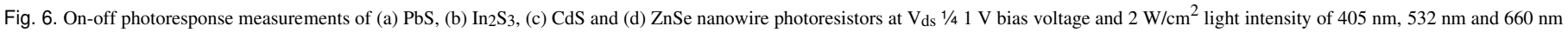
wavelength light illumination. The diameters of $\mathrm{PbS}, \mathrm{In}_{2} \mathrm{~S}_{3}, \mathrm{CdS}$ and $\mathrm{ZnSe} \mathrm{NWs}$ are 120, 160, 180 and $280 \mathrm{~nm}$, respectively.

Table 1

Comparison of the photoresponses of the studied nanowire-based photoresistors

\begin{tabular}{|c|c|c|c|c|c|}
\hline \multirow[t]{2}{*}{ Materials } & \multirow[t]{2}{*}{$\mathrm{Eg}_{\mathrm{g}}(\mathrm{eV})$} & \multirow[t]{2}{*}{$\mathrm{I}_{\text {dark }}$ at $1 \mathrm{~V}(\mathrm{nA})$} & \multicolumn{3}{|l|}{$\mathrm{I}_{\mathrm{on}} / \mathrm{I}_{\text {off }}$ ratio } \\
\hline & & & at $405 \mathrm{~nm}(3.06 \mathrm{eV})$ & at $532 \mathrm{~nm}(2.33 \mathrm{eV})$ & at $660 \mathrm{~nm}(1.88 \mathrm{eV})$ \\
\hline $\mathrm{PbS}$ NW & 0.41 & 2.65 & 1.11 & 1.07 & 1.03 \\
\hline $\mathrm{In}_{2} \mathrm{~S}_{3} \mathrm{NW}$ & 2.1 & 9.95 & 11.3 & 2.8 & 2.1 \\
\hline CdS NW & 2.4 & 3.65 & 2.7 & 1.4 & 1.1 \\
\hline ZnSe NW & 2.7 & 0.02 & 115 & 1 & 1 \\
\hline
\end{tabular}

Table 2

Responsivity R/ and external quantum efficiency EQE values of the studied nanowire-based photoresistors at different illumination wavelengths.

\begin{tabular}{|c|c|c|c|c|c|c|}
\hline \multirow[t]{2}{*}{ Materials } & \multicolumn{3}{|l|}{$\mathrm{R} \mid, \mathrm{A} / \mathrm{W}$} & \multicolumn{3}{|l|}{$\mathrm{EQE}$} \\
\hline & at $405 \mathrm{~nm}$ & at $532 \mathrm{~nm}$ & at $660 \mathrm{~nm}$ & at $405 \mathrm{~nm}$ & at $532 \mathrm{~nm}$ & at $660 \mathrm{~nm}$ \\
\hline $\mathrm{PbS}$ NW & 0.06 & 0.04 & 0.02 & $18 \%$ & $9 \%$ & $3 \%$ \\
\hline $\mathrm{In}_{2} \mathrm{~S}_{3} \mathrm{NW}$ & 16.01 & 2.80 & 1.71 & $4903 \%$ & $652 \%$ & $321 \%$ \\
\hline CdS NW & 0.86 & 0.20 & 0.05 & $264 \%$ & $47 \%$ & $10 \%$ \\
\hline ZnSe NW & 0.20 & 0 & 0 & $62 \%$ & 0 & 0 \\
\hline
\end{tabular}

specific NW current signals, which arise from distinct photo-response values of NWs [38,39]. However, this above-cut-off-wavelength photoresponse might be considerably reduced by sur-face passivation, therefore simplifying the principle of colour distinction. That could be done by producing a shell layer around the core material that eliminates its surface trap states, and could even lead to an enhanced photosensitivity [40e42]. In general, choosing different photosensitive NWs for desired spectral range, depending on the application, is possible yet not always sufficient e defects, such as NW surface, or impurity doping can result in above-cut-off-wavelength photosensitivity.

\section{Conclusions}

$\mathrm{PbS}, \mathrm{In}_{2} \mathrm{~S}_{3}, \mathrm{CdS}$ and $\mathrm{ZnSe} \mathrm{NWs}$ were synthesised via atmospheric pressure chemical vapour transport method. Samples were char-acterized by SEM, TEM methods, XRD technique, which revealed their highly crystalline structure, and PL measurements to inves-tigate their optical properties. Twoterminal photoresistor devices were fabricated from as-grown NWs using in situ nano-manipulations and electron-beam-assisted Pt deposition inside SEM-FIB; their current-voltage and photoresponse measurements were performed under the same conditions. Two approaches for 
determining the colour (red, green or blue) with as-prepared NW photoresistors were discussed. While determination via the cut-off wavelength due to the bandgap would be the best approach in this case, considerable above-cut-off-wavelength photosensitivity was observed; therefore, a method for reducing this undesired photo-response is necessary to be implemented.

\section{Acknowledgements}

Financial support provided by Scientific Research Project for Students and Young Researchers Nr. SJZ/2016/6 realized at the Institute of Solid State Physics, University of Latvia is greatly acknowledged. Authors are grateful to Reinis Ignatans for XRD measurements.

\section{References}

[1] E.H. Steenbergen, M.J. DiNezza, W.H.G. Dettlaff, S.H. Lim, Y.-H. Zhang, Opti-callyaddressed two-terminal multicolor photodetector, Appl. Phys. Lett. 97 (2010) 161111, https://doi.org/10.1063/1.3505137.

[2] G. Konstantatos, E.H. Sargent, Nanostructured materials for photon detection, Nat. Nanotechnol. 5 (2010) 391e400, https://doi.org/10.1038/nnano.2010.78.

[3] Y. Liu, R. Cheng, L. Liao, H. Zhou, J. Bai, G. Liu, L. Liu, Y. Huang, X. Duan, Plasmon resonance enhanced multicolour photodetection by graphene, Nat. Commun. 2 (2011) 579, https://doi.org/10.1038/ncomms1589.

[4] E. Laux, C. Genet, T. Skauli, T.W. Ebbesen, Plasmonic photon sorters for spectral and polarimetric imaging, Nat. Photonics 2 (2008) 161e164, https:// doi.org/10.1038/nphoton.2008.1.

[5] C. Soci, A. Zhang, B. Xiang, S.A. Dayeh, D.P.R. Aplin, J. Park, X.Y. Bao, Y.H. Lo, D. Wang, ZnO nanowire UV photodetectors with high internal gain, Nano Lett. 7 (2007) 1003e1009, https://doi.org/10.1021/nl070111x.

[6] K. Deng, L. Li, CdS nanoscale photodetectors, Adv. Mater. 26 (2014) 2619e2635, https://doi.org/10.1002/adma.201304621.

[7] R. Graham, C. Miller, E. Oh, D. Yu, Electric field dependent photocurrent decay length in single lead sulfide nanowire field effect transistors, Nano Lett. 11 (2011) 717e722, https://doi.org/10.1021/nl1038456.

[8] X. Xie, G. Shen, Single-crystalline In2S3 nanowire-based flexible visible-light photodetectors with an ultra-high photoresponse, Nanoscale 7 (2015) 5046e5052, https://doi.org/10.1039/C5NR00410A.

[9] C.H. Hsiao, S.J. Chang, S.B. Wang, S.P. Chang, T.C. Li, W.J. Lin, C.H. Ko, T.M. Kuan, B.R. Huang, ZnSe nanowire photodetector prepared on oxidized silicon sub-strate by molecular-beam epitaxy, J. Electrochem. Soc. 156 (2009), https:// doi.org/10.1149/1.3077580. J73.

[10] T. Takahashi, P. Nichols, K. Takei, A.C. Ford, A. Jamshidi, M.C. Wu, C.Z. Ning, A. Javey, Contact printing of compositionally graded $\mathrm{CdSxSe} 1-\mathrm{x}$ nanowire parallel arrays for tunable photodetectors, Nanotechnology 23 (2012) 45201, https://doi.org/10.1088/0957-4484/23/4/045201.

[11] H. Dedong, L. Ying-Kai, D. Yu, Multicolor photodetector of a single Er3p-Doped CdS nanoribbon, Nanoscale Res. Lett. 10 (2015) 285, https://doi.org/ 10.1186/s11671-0150975-3.

[12] H. Park, Y. Dan, K. Seo, Y.J. Yu, P.K. Duane, M. Wober, K.B. Crozier, Filter-free image sensor pixels comprising silicon nanowires with selective color ab-sorption, Nano Lett. 14 (2014) 1804e1809, https://doi.org/10.1021/nl404379w.

[13] X. Liu, L. Jiang, X. Zou, X. Xiao, S. Guo, C. Jiang, X. Liu, Z. Fan, W. Hu, X. Chen, W. Lu, W. Hu, L. Liao, Scalable integration of indium zinc oxide/ photosensitivenanowire composite thin-film transistors for transparent multicolor photodetectors array, Adv. Mater. 26 (2014) 2919e2924, https:// doi.org/10.1002/adma.201305073.

[14] L. Sang, J. Hu, R. Zou, Y. Koide, M. Liao, Arbitrary multicolor photodetection by heterointegrated semiconductor nanostructures, Sci. Rep. 3 (2013) 2368, https://doi.org/10.1038/srep02368.

[15] Z. Fan, J.C. Ho, T. Takahashi, R. Yerushalmi, K. Takei, A.C. Ford, Y.-L. Chueh, A. Javey, Toward the development of printable nanowire electronics and sensors, Adv. Mater. 21 (2009) 3730e3743, https://doi.org/10.1002/ adma.200900860.

[16] S.Y. Jang, Y.M. Song, H.S. Kim, Y.J. Cho, Y.S. Seo, G.B. Jung, C. Lee, J. Park, M. Jung, J. Kim, B. Kim, J.-G. Kim, Y.-J. Kim, Three synthetic routes to singlecrystalline $\mathrm{PbS}$ nanowires with controlled growth direction and their elec-trical transport properties, ACS Nano 4 (2010) 2391e2401, https://doi.org/ 10.1021/nn100163k.

[17] M. Zervos, P. Papageorgiou, A. Othonos, High yieldelow temperature growth of indium sulphide nanowires via chemical vapor deposition, J. Cryst. Growth 312 (2010) 656e661, https://doi.org/10.1016/j.jcrysgro.2009.12.023.

[18] Y. Wang, G. Meng, L. Zhang, C. Liang, J. Zhang, Catalytic growth of large-scale singlecrystal CdS nanowires by physical evaporation and their photo-luminescence, Chem Mater. 14 (2002) 1773e1777, https://doi.org/10.1021/ cm0115564.

[19] U. Philipose, P. Sun, T. Xu, H.E. Ruda, L. Yang, K.L. Kavanagh, Structure and photoluminescence of $\mathrm{ZnSe}$ nanostructures fabricated by vapor phase growth, J. Appl. Phys. 101 (2007) 14326, https://doi.org/10.1063/1.2424400.

[20] A. Datta, G. Sinha, S.K. Panda, A. Patra, Growth, optical, and electrical prop-erties of In2S3 zigzag nanowires, Cryst. Growth Des. 9 (2009) 427e431, https://doi.org/10.1021/cg800663t.

[21] L. Dong, J. Jiao, M. Coulter, L. Love, Catalytic growth of CdS nanobelts and nanowires on tungsten substrates, Chem. Phys. Lett. 376 (2003) 653e658, https://doi.org/10.1016/S0009-2614(03)01059-5.

[22] S. Kar, S. Biswas, S. Chaudhuri, Catalytic growth and photoluminescence properties of ZnS nanowires, Nanotechnology 16 (2005) 737e740, https:// doi.org/10.1088/09574484/16/6/018

[23] Y.K. Albert Lau, D.J. Chernak, M.J. Bierman, S. Jin, Epitaxial growth of hierar-chical PbS nanowires, J. Mater. Chem. 19 (2009) 934, https://doi.org/10.1039/ b818187j.

[24] JCPDS Card No. 78e1058, (n.d.)

[25] JCPDS Card No. 25e0390, (n.d.)

[26] JCPDS Card No. 21e829, (n.d.)

[27] JCPDS Card No. 37e1463, (n.d.)

[28] JCPDS Card No. 41e1049, (n.d.)

[29] N.S. Dantas, A.F. da Silva, C. Persson, Electronic band-edge properties of rock salt PbY and $\mathrm{SnY}\left(\mathrm{Y}^{1 / 1} / 4\right.$ S, Se, and Te), Opt. Mater. (Amst) 30 (2008) 1451e1460, https://doi.org/10.1016/j.optmat.2007.09.001.

[30] A.D. Andreev, A.A. Lipovskii, Anisotropy-induced optical transitions in $\mathrm{PbSe}$ and $\mathrm{PbS}$ spherical quantum dots, Phys. Rev. B 59 (1999) 15402e15404, https://doi.org/10.1103/PhysRevB.59.15402.

[31] H. Cao, G. Wang, S. Zhang, X. Zhang, Growth and photoluminescence prop-erties of PbS nanocubes, Nanotechnology 17 (2006) 3280e3287, https:// doi.org/10.1088/09574484/17/13/034

[32] T. Tohidi, K. Jamshidi-Ghaleh, Optical and structural properties of nano-crystalline $\mathrm{PbS}$ thin film grown by CBD on $\operatorname{Si}\left(\begin{array}{lll}1 & 0 & 0\end{array}\right)$ substrate, Philos. Mag. 94 (2014) 3368e3381, https://doi.org/10.1080/14786435.2014.959579.

[33] A.A. Talin, F. Leonard, B.S. Swartzentruber, X. Wang, S.D. Hersee, Unusually strong space-charge-limited current in thin wires, Phys. Rev. Lett. 101 (2008) 76802, https://doi.org/10.1103/PhysRevLett.101.076802.

[34] A.M. Katzenmeyer, F. Leonard, A.A. Talin, M.E. Toimil-Molares, J.G. Cederberg, J.Y. Huang, J.L. Lensch-Falk, Observation of space-charge-limited transport in InAs nanowires, IEEE Trans. Nanotechnol. 10 (2011) 92e95, https://doi.org/ 10.1109/TNANO.2010.2062198.

[35] A. Vila, F. Hernandez-Ramirez, J. Rodríguez, O. Casals, A. Romano-Rodríguez, J.R. Morante, M. Abid, Fabrication of metallic contacts to nanometre-sized materials using a focused ion beam (FIB), Mater. Sci. Eng. C 26 (2006) 1063e1066, https://doi.org/10.1016/j.msec.2005.09.092.

[36] Y. Ye, L. Dai, X. Wen, P. Wu, R. Pen, G. Qin, High-performance single CdS nanobelt metal-semiconductor field-effect transistor-based photodetectors, ACS Appl. Mater. Interfaces 2 (2010) 2724e2727, https://doi.org/10.1021/ am100661x.

[37] T. Zhai, L. Li, X. Wang, X. Fang, Y. Bando, D. Golberg, Recent developments in onedimensional inorganic nanostructures for photodetectors, Adv. Funct. Mater. 20 (2010) 4233e4248, https://doi.org/10.1002/adfm.201001259.

[38] Y.-Q. Bie, Z.-M. Liao, H.-Z. Zhang, G.-R. Li, Y. Ye, Y.-B. Zhou, J. Xu, Z.-X. Qin, L. Dai, D.-P. Yu, Self-powered, ultrafast, visible-blind UV detection and optical logical operation based on $\mathrm{ZnO} / \mathrm{GaN}$ nanoscale p-n junctions, Adv. Mater. 23 (2011) 649e653, https://doi.org/10.1002/adma.201003156.

[39] Z. Fan, J.C. Ho, Z.A. Jacobson, H. Razavi, A. Javey, Large-scale, heterogeneous integration of nanowire arrays for image sensor circuitry, Proc. Natl. Acad. Sci. 105 (2008) 11066e11070, https://doi.org/10.1073/pnas.0801994105.

[40] L. Qin, C. Shing, S. Sawyer, P.S. Dutta, Enhanced ultraviolet sensitivity of zinc oxide nanoparticle photoconductors by surface passivation, Opt. Mater. (Amst) 33 (2011) 359e362, https://doi.org/10.1016/j.optmat.2010.09.020.

[41] K. Moazzami, T.E. Murphy, J.D. Phillips, M.C.-K. Cheung, A.N. Cartwright, Subbandgap photoconductivity in $\mathrm{ZnO}$ epilayers and extraction of trap density spectra, Semicond. Sci. Technol. 21 (2006) 717e723, https://doi.org/10.1088/ 02681242/21/6/001

[42] Y. Dan, K. Seo, K. Takei, J.H. Meza, A. Javey, K.B. Crozier, Dramatic reduction of surface recombination by in situ surface passivation of silicon nanowires, Nano Lett. 11 (2011) 2527e2532, https://doi.org/10.1021/nl201179n. 